\title{
Imbalance between Pneumocystis carinii cysts and trophozoites in bronchoalveolar lavage fluid from patients with pneumocystosis receiving prophylaxis
}

\author{
ENRICA TAMBURRINI, PAOLA MENCARINI, ELENA VISCONTI, A. DE LUCA, MARIA ZOLFO, \\ ALESSANDRA SIRACUSANO*, ELENA ORTONA*, RITA MURRI and A. ANTINORI \\ Department of Infectious Diseases, Università Cattolica S. Cuore, Rome and *Department of Immunology, \\ Istituto Superiore di Sanità, Rome, Italy
}

\begin{abstract}
Detection and quantification of different Pneumocystis carinii (PC) life cycle forms were performed by polymerase chain reaction (PCR) and by morphological stains on bronchoalveolar lavage fluids (BALF) from HIV-infected patients with $P$ carinii pneumonia (PCP). The number of PC trophozoites was higher in patients with PCP who were receiving prophylaxis than in those not receiving prophylaxis. Also the cyst: trophozoite ratio was lower in the first group. No difference was observed between patients receiving different prophylactic medications. The imbalance between $P C$ forms in BALF from patients with PCP receiving anti-PC prophylaxis may hamper the sensitivity of cyst stains. Multiple stains or PCR examination should be performed on BALF from patients with clinically suspected PCP who are receiving prophylaxis.
\end{abstract}

\section{Introduction}

Bronchoalveolar lavage fluid (BALF) and induced sputum (IS) examination for Pneumocystis carinii (PC) were reported to yield a significantly lower diagnostic sensitivity in HIV-infected patients with PCP receiving aerosolised pentamidine as anti-PC prophylaxis $[1,2]$. A clear mechanism for the decreased yield of diagnostic procedures in these patients was not identified. Other studies reported that prophylactic treatment with aerosolised pentamidine had no effect on the diagnostic sensitivity of PC detection in IS or BALF, or on the number and the different life cycle forms of the organisms detected in BALF of HIVinfected patients with PCP $[3,4]$.

To further investigate the influence of anti-PC prophylaxis on the organism load, the cyst:trophozoite ratio and the PC DNA content were assessed in BALF from $\mathrm{HIV}$-infected patients experiencing a first episode of pneumocystosis while receiving prophylaxis and in patients not receiving prophylaxis.

\section{Patients and methods \\ Patients and clinical specimens}

Twenty-one consecutive HIV-infected patients presenting with a first episode of PCP (19 males, two females;

Received 17 Oct. 1995; revised version accepted 18 Dec. 1995.

Corresponding author: Dr E. Tamburrini. age 26-51 years) observed at the Clinic of Infectious Diseases, Università Cattolica S. Cuore, Rome, between 1 Jan. and 31 Dec. 1994 were studied. Seven patients were receiving anti-PC primary oral prophylaxis with dapsone (50 $\mathrm{mg}$ daily) plus pyrimethamine (50 mg weekly); six patients were taking aerosolised pentamidine ( $300 \mathrm{mg}$ every 4 weeks); and eight patients, previously unaware of being HIV-infected, were receiving no prophylaxis.

Bronchoalveolar lavage was performed before starting specific therapy by two different operators who used the same technique with 'multiple lobe' sampling. BALF samples were processed immediately for morphological examination for $\mathrm{PC}$ and a sample was stored at $-20^{\circ} \mathrm{C}$ for PCR.

\section{Detection and quantification of PC organisms}

The presence of PC organisms was detected by staining cysts and trophozoites. Indirect immunofluorescence with monoclonal antibodies specific for antigens of the cyst wall (Monofluokit P. carinii, Diagnostics Pasteur, Paris) was performed according to the manufacturer's instructions. Giemsa staining was performed by a standard protocol.

For diagnostic purposes, two lavage slides were examined carefully by two investigators.

To quantify the number of organisms in BALF, two $10-\mu 1$ drops of clinical samples were placed on a glass 
slide covering an area of c. $1 \mathrm{~cm}^{2}$. A total of 20 oil immersion fields was randomly scanned. The total count in $1.0 \mathrm{ml}$ was calculated according to the formula reported by Bartlett et al. [5]. To minimise the miscounting of trophozoites, the results were also expressed as the cyst:trophozoite ratio. In addition, the percentage of nuclei/cysts and the total number of PC nuclei in $1 \mathrm{ml}$ of BALF were estimated.

\section{PC DNA quantification by PCR}

Quantification of PC organisms was also performed by amplifying DNA with primers specific for the mitochondrial large subunit rRNA in serial endpoint dilutions of lavage fluids. Briefly, samples of BALF were digested with proteinase $\mathrm{K}$ for $2 \mathrm{~h}$ at $56^{\circ} \mathrm{C}$, extracted with phenol:chloroform, diluted and amplified with pAZ102 E and H [6]. PCR products were electrophoresed in agarose $2 \%$ gel and stained by ethidium bromide. DNA extracted from BALF was serially diluted to 1 in 20000 . All dilution experiments were performed in duplicate. Multiple negative and positive controls were included in each amplification run.

\section{Results}

On the basis of clinical data, response to treatment and morphological identification of PC, all the patients were found to have PCP.

The three groups had similar clinical and immunological characteristics. In particular, they were similar with respect to the severity of pneumonia as expressed by the alveolar-arterial ( $\mathrm{A}-\mathrm{a})$ oxygen gradient, and to the immunological status as expressed by the absolute $\mathrm{CD}^{+}$count. Moreover, the two groups on prophylaxis were similar with respect to the duration of having less than $200 \times 10^{6} / \mathrm{L} \mathrm{CD}^{+}$cells.

P. carinii organisms were detected by both IF and Giemsa stains and PC DNA was detected by PCR in BALF from all the patients.

Results of PC quantification are shown in Table 1.

\section{Discussion}

The results show that patients with a PCP episode whilst receiving prophylaxis display an imbalance between the different life cycle forms of PC. No difference was observed between patients who received dapsone plus pyrimethamine or aerosolised pentamidine.

PCR quantification showed that the number of PC nuclei detectable in BALF from patients with PCP was similar, whether or not they were receiving prophylaxis. These data are in agreement with those reported by $\mathrm{Ng}$ et al. [4] on induced sputum or BALF from patients treated with aerosolised pentamidine. Their laboratory method was based on the detection of clumps that contained cysts or trophozoites, or both.

The finding of an increased number of tropozoites associated with a decreased number of PC cysts without a change in the total PC organism burden in patients receiving prophylaxis explains the conflicting diagnostic results of previous reports [1,4]. Indeed, different methods of PC quantification were used, based either on cyst wall staining [1] or on organism nuclei stains [4].

Although the relative importance of PC cysts and trophozoites in the infected lung has not been established definitely, it is generally assumed that the two forms are interdependent. Recently it has been reported that the cyst stage is an obligatory step in trophozoite proliferation [7]. The visualisation of characteristic cysts in respiratory secretions is considered diagnostic [8]. However, Elvin et al. [9] reported a PCP case with free trophozoites and very few cysts in BALF. These results suggested that some cases of PCP could be missed if the diagnosis of active infection depended on the visualisation of cysts only.

The reasons for this peculiar pattern of PC organisms in BALF from HIV patients receiving prophylaxis are not completely clear. Patients not receiving prophylaxis were similar to those on prophylaxis with respect

Table 1. P. carinii in BALF from patients with PCP: comparison between patients receiving prophylaxis with dapsone pyrimethamine or aerosolised pentamidine and those who never received prophylaxis

\begin{tabular}{|c|c|c|c|c|c|}
\hline \multirow{2}{*}{$\begin{array}{l}\text { Patient } \\
\text { group }\end{array}$} & \multicolumn{3}{|c|}{ Quantification of $\mathrm{PC}^{*}$} & \multirow{2}{*}{$\begin{array}{c}\text { Estimated } \\
\text { organisms } \\
(\mathrm{PC} \text { nuclei } / \mathrm{ml})^{*}\end{array}$} & \multirow{2}{*}{$\begin{array}{l}\text { Cyst: trophozoite } \\
\text { ratio (median) } \S\end{array}$} \\
\hline & $\mathrm{PCR} \dagger$ & Cysts $/ \mathrm{ml}$ & Trophozoites/ml $\ddagger$ & & \\
\hline No prophylaxis & $\begin{array}{l}1077 \\
(348)\end{array}$ & $\begin{array}{r}125856 \\
(89997)\end{array}$ & $\begin{array}{r}427612 \\
(65764)\end{array}$ & $\begin{array}{l}1057000 \\
(468310)\end{array}$ & $1: 10$ \\
\hline $\begin{array}{l}\text { Dapsone }+ \\
\text { pyrimethamine }\end{array}$ & $\begin{array}{r}971 \\
(364)\end{array}$ & $\begin{array}{r}30945 \\
(18348)\end{array}$ & $\begin{array}{l}1242000 \\
(273282)\end{array}$ & $\begin{array}{l}1397000 \\
(319634)\end{array}$ & $1: 121$ \\
\hline $\begin{array}{l}\text { Aerosolised } \\
\text { pentamidine }\end{array}$ & $\begin{array}{l}1070 \\
(418)\end{array}$ & $\begin{array}{l}16425 \\
(5445)\end{array}$ & $\begin{array}{l}2074000 \\
(788524)\end{array}$ & $\begin{array}{l}2156000 \\
(197541)\end{array}$ & $1: 198$ \\
\hline
\end{tabular}

${ }^{*}$ Results are expressed as mean (SEM)

$\uparrow$ Maximal positive dilution

$\ddagger \mathrm{p}=0.018 ; \S \mathrm{p}=0.009$ 
to immunological parameters and clinical severity of PCP.

The pattern of PC organisms in BALF from patients on prophylaxis was not associated with continuous contact with PC sources, i.e., other HIV patients with $\mathrm{PCP}$ or patients receiving anti-PC primary prophylaxis (data not shown).

These results suggest that dapsone plus pyrimethamine and aerosolised pentamidine may interfere with the life cycle of PC through inhibition of replication of cystic forms.

In conclusion, the imbalance between the different life cycle forms of PC with an increased number of trophozoites could explain the lower diagnostic yield of the cyst-staining immunofluorescence in BALF and induced sputum from patients receiving prophylaxis. We believe that multiple stains or PCR examination should be performed on BALF from patients receiving anti-PC prophylaxis if PCP is suspected clinically.

This work was supported by a grant from the AIDS Project (1995, n.930Q), Istituto Superiore di Sanità, Ministero della Sanità, Roma and by a grant from the European Concerted Action (Contract $n$. BMH1-CT94-1118)

\section{References}

1. Jules-Elysee KM, Stover DE, Zaman MB, Bernard EM, White DA. Aerosolized pentamidine: effect on diagnosis and presentation of Pneumocystis carinii pneumonia. Ann Intern Med 1990; 112: $750-757$.

2. Levine SJ, Masur $\mathrm{H}$, Gill VJ et al. Effect of aerosolized pentamidine prophylaxis on the diagnosis of Pneumocystis carinii pneumonia by induced sputum examination in patients infected with the Human Immunodeficiency Virus. Am Rev Respir Dis 1991; 144: 760-764.

3. Fahy JV, Chin DP, Schnapp LM et al. Effect of aerosolized pentamidine prophylaxis on the clinical severity and diagnosis of Pneumocystis carinii pneumonia. Am Rev Respir Dir 1992; 146: $844-848$

4. Ng VL, Geaghan SM, Leoung G et al. Lack of effect of prophylactic aerosolized pentamidine on the detection of Pneumocystis carinii in induced sputum or bronchoalveolar lavage specimens. Arch Pathol Lab Med 1993; 117: 493-496.

5. Bartlett MS, Verbanac PA, Smith JW. Cultivation of Pneumocystis carinii with WI-38 cells. J Clin Microbiol 1979; 10: 796799.

6. Wakefield AE, Pixley FJ, Banerji $\mathrm{S}$ et al. Detection of Pneumocystis carinii with DNA amplification. Lancet 1990; 336: $451-453$.

7. Schmatz DM, Powles MA, McFadden DC et al. Antipneumocystis activity of water-soluble lipopeptide L-693, 989 in rats. Antimicrob Agents Chemother 1992; 36: 1964-1970.

8. Gill VJ, Evans G, Stock F, Parrillo JE, Masur H, Kovacs JA Detection of Pneumocystis carinii by fluorescent-antibody stain using a combination of three monoclonal antibodies. $J$ Clin Microbiol 1987; 25: 1837-1840.

9. Elvin KM, Björkman A, Linder E, Heurlin N, Hierpe A. Pneumocystis carinii pneumonia: detection of parasites in sputum and bronchoalveolar lavage fluid by monoclonal antibodies. BMJ 1988; 297: 381-384. 